\title{
User-Personality Classification Based on the Non-Verbal Cues from Spoken Conversations
}

\author{
Soonil Kwon ${ }^{1}$, Joon Yeon Choeh ${ }^{2}$ Jong-Weon Lee ${ }^{3}$ \\ ${ }^{1}$ Department of Digital Contents, Sejong University, \\ 98 Gunjs-dong, Gwangjin-gu, Seoul, 143-737, Korea \\ E-mail:skwon@sejong.edu \\ ${ }^{2}$ Department of Digital Contents, Sejong University, \\ 98 Gunjs-dong, Gwangjin-gu, Seoul, 143-737, Korea \\ E-mail:zoon@sejong.ac.kr \\ ${ }^{3}$ Department of Digital Contents, Sejong University, \\ 98 Gunjs-dong, Gwangjin-gu, Seoul, 143-737, Korea \\ E-mail: jwlee@sejong.ac.kr
}

\begin{abstract}
Technology that detects user personality based on user speech signals must be researched to enhance the function of interaction between a user and virtual agent that takes place through a speech interface. In this study, personality patterns were automatically classified as either extroverted or introverted. Personality patterns were recognized based on non-verbal cues such as the rate, energy, pitch, and silent intervals of speech with patterns of their change. Through experimentation, a maximum pattern classification accuracy of $86.3 \%$ was achieved. Using the same data, another pattern classification test was manually carried out by people to see how well the automatic pattern classification of personal traits performed. The results in the second manual test showed an accuracy of $86.6 \%$. This proves that the automatic pattern classification of personal traits can achieve results comparable to the level of performance accomplished by humans. The Silent Intervals feature of the automatic pattern classification performed admirably while in the second test done by people, pitch was a key factor in producing better accuracy. This information will be useful and applicable in future studies.
\end{abstract}

Keywords: Voice User Interface, User Personality Trait, Speech Processing, Human-Computer Interaction.

\section{Introduction}

The functional content market, fueled by the robotics, gaming, and education industries, is experiencing rapid growth with each passing year. This market not only has industrial merit but also is a sub- ject well worth researching. Currently, there are still not enough sources and application technologies related to interfaces used in functional contents. To address this issue and advance the functional content market along with related industries, focused research on innovative interfaces and their application

${ }^{*}$ Corresponding author 
to content production must be undertaken. . $2,3,4^{2,4}$

To provide the user with user-oriented intelligent services, a technology that identifies and processes human thoughts, personality traits, and emotions for efficient and accurate recognition of user features is required. User speech signals contain not only linguistic meanings but also elements of the emotions which are the features of the speaker. Research in emotion recognition from speech has been taking place for decades and is expected to break new ground in the future. However, it must be further honed as the expression of emotions tends to be more subjective than objective. In contrast to the volatility of emotions, user personalities tend to be consistent, and the inherent features they present can be analyzed to identify user features.

The aim of this research is to propose an approach for automatic personality trait classification based on non-verbal behavioral cues characterizing the way someone speaks, as well as to gain a better understanding of user personality traits by analyzing user speech information. In the middle of preliminary studies, we took much interest in both extroversion and introversion, though there are various kind of personality traits, since this pair of traits is usually perceived more quickly and accurately by people ${ }^{5}$ and computational devices. In addition, it is supposed to be useful for the purpose of developing a core technology to be applied to foreign language learning contents (provision of contents to suit different users) that will be a final target application in our ongoing work. 6,7

To achieve automatic personality trait classification, a variety of non-verbal cues in speech signals, such as the changes in the speed, energy, and pitch of speech, were identified and analyzed based on existing analytical and cognitive psychology research. In this paper, silent intervals, such as the length, proportion, and distribution of silent intervals, were mainly taken into consideration for the roles and validity of user personality patterns.

Source technology developed based on this research can be effectively applied to various types of e-learning contents as well as systems that require user feature information such as robot dialog systems and automated responses to consumer in- quiries. It can also be applied to functional contents designed for the psychological treatment and rehabilitation of individuals.

The paper is organized as follows: section 2 outlines and analyzes existing research related to this research; section 3 outlines the feature vectors in voice information used to estimate user personality traits and how they are extracted; section 4 outlines a pattern classification method based on the feature vectors extracted in section 3 used to determine user personalities; section 5 outlines experimental processes and outcomes; section 6 describes a supplementary experiment and discusses its result supporting our approach; and, section 7 provides the conclusion of this research.

\section{Related Work}

Analytical psychologist Carl Gustav Jung classified personalities as extroverted and introverted based on the type of conscious attitude exhibited by an individual's personality. Conscious attitude is determined by an individual's mental energy. If an individual's mental energy is externally oriented, the individual is classified as having an extroverted attitude; if an individual's mental energy is internally oriented, the individual is classified as having an introverted attitude. In other words, personalities are classified as extroverted or introverted depending on whether their mental energies are externally oriented or internally oriented. ${ }^{8}$

J. Dewaele and A. Furnham researched the influence of an individual's personality on the use of mother tongue (L1) and a second language (L2), and discovered that extroverted individuals had a better command of L2 while introverted individuals were hesitant and not fluent in the use of L2. ${ }^{6}$ Although this study was related to the fluency of L2, it also showed the promise of extroversion/introversion classification based on voice data extracted from conversations.

A. Furnham et al. researched the correlation of personality and intelligence in the field of academics. Extroverted individuals were found to be fluent, but their speech was marked by many mistakes. The conclusion was reached that tools rather 
than language would be better used in intelligence assessments of extroverted individuals. On the other hand, introverted individuals were more thoughtful in their speech, used a more refined vocabulary, and made fewer mistakes, leading to the conclusion that language could be effectively used in intelligence assessments of introverted individuals. ${ }^{9}$ From this study, we confirmed that silent intervals and slow speech, which is a sign of thoughtfulness, are likely pronounced among introverted types. These factors are necessary data for extroversion/introversion classification.

A. J. Gill and J. Oberlander researched how an individual's personality influences an individual's public speaking performance by analyzing a bi-gram of writing prepared using corpus linguistics and categorizing it into eight features. They found that extroverted individuals used economical language and did not talk about themselves. They also found that extroverted individuals used a far greater number of words (with a tendency to use positive words) than introverted individuals and maintained an open attitude. In contrast, introverted individuals did not address the future, did not use the word "we," and tended to use negative words frequently. ${ }^{10}$ This study mainly focused on linguistic analysis and interpretation while lacked an in-depth look into nonverbal cues. We realized the need for further investigation.

K. R. Scherer and U. Scherer studied the general public, jurors, and government employees to define the features of extroverted and introverted personalities. During interviews, extroverted subjects were more at ease than introverted subjects and also spoke more frequently and more loudly. ${ }^{11}$ An experiment conducted by K. R. Scherer also proved that the voices of extroverted individuals had higher pitches and wider sound change ranges. ${ }^{12}$ This study showed that the decibel range and energy expelled by test subjects play an integral role in extroversion/introversion classification. There was a realization among us that the change in pitch is more important than the absolute pitch numbers.

W. Apple et al. experimentally proved that listeners use acoustic properties in making personal attributions to speakers. Speakers with high-pitched voices were judged less truthful, less emphatic, less potent (smaller, thinner, faster) and more nervous. Slow-talking speakers were judged less truthful, less fluent, and less persuasive, and were seen as more passive (slower, colder, passive, weaker) but more potent. ${ }^{13}$ This study provided raw data proving that the pitch and speed of a person's voice plays a critical role in classifying personality traits.

Although there is a variety of existing studies, research on the sound elements of speech is quantitatively and qualitatively insufficient. Speech features that have been researched so far mainly include the speed, energy, and pitch of speech. Moreover, no indepth studies exist on how well such speech features would be recognized when applied to an automated (computerized) personality classification system.

In contrast to the significant amount of previous research, we describe an automated system for personality prediction in the context of spoken conversations. In our research, only non-verbal cues exhibited in a user's speech have been used to classify the user personality traits: extroversion/introversion. Those cues are supposed to be manifested in nonverbal cues extracted from speech signals. In addition, they could be technologically measured and assessed for pattern recognition.

\section{Personality Trait Classification and Non-Verbal Cues}

The personality trait of a user is expressed through the user's various behaviors. In light of existing research conducted by a number of researchers, extroverted and introverted individuals exhibit a difference in vocabulary selection and sentence structuring. However, to analyze linguistic information, a speech recognition engine in company with a natural language processing unit is required which could be a burden of computation and memory to small and mobile devices. In this study, without a speech recognition and natural language processing unit, non-verbal cues of exhibited in user speech were analyzed to determine the personality trait of a computer user through a voice interface. In other words, we made an algorithm to classify a user personality trait based on the speech uttered by the user includ- 
ing the manner of speaking.

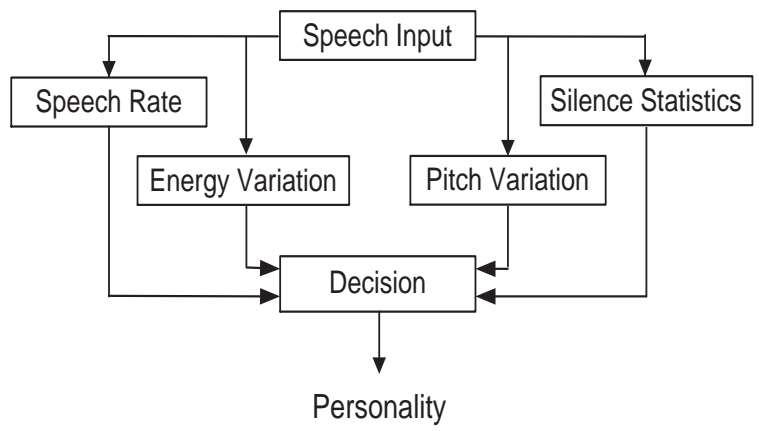

Fig. 1. Block diagram of user personality classification. ${ }^{9,11,12,13}$

Previous studies have shown that the extroverted tend to talk more frequently, more quickly, and more loudly than the introverted. ${ }^{8,9,11}$ From those studies, we assumed that the common reason of the above phenomenon is originated from the relationship between speaking and thinking. The introverted tend to think what they are going to say beforehand as well as in the middle of speaking compared with the extroverted. From this hypothesis, we applied a new feature, a silent interval, to user personality trait classification. Figure 1 shows the utilization for user personality classification. We mainly extracted four kinds of information from the speech: speech rate, the energy of speech, the fundamental frequency related with pitch, and silence statistics such as absolute length of silence, the proportion of silence in total speech, the frequency of long silence, the length of intervals between the end of a question, and the beginning of an answer.

Speech rate (speed of talking) is a measurement of how many words or syllables are spoken within a time unit. While it is difficult to quantify how much an individual talks, talking speed can be measured by counting how many syllables and words are spoken in a set talk duration. ${ }^{14}$ In this study, only the non-linguistic cues in speech signals were measured to determine speech rate without assessing linguistic information. Hence, speech rates are measured as follows based on the enrate system presented by Nelson Morgan ${ }^{15}$ :
1. Half-wave rectified input signals are passed through a low-pass filter and down-sampled in $100 \mathrm{~Hz}$.

2. 1- to 2-second windows overlapped by more than $75 \%$ are applied to the signals.

3. A short-term spectrum is obtained through the discrete Fourier transform (DFT).

4. Spectral moment is calculated.

It has also been found that the voices of the extroverted are generally louder and higher-pitched ${ }^{11,12}$; this can be determined by observing the change in the average energy and pitch of speech signals for a set duration. Energy can be easily calculated through spectrum analysis. However, the absolute value of energy of individuals with loud voices is likely to be high and cannot be reliably used to determine extroverted and introverted personalities. Therefore, the relative rate of change in energy, rather than an absolute value of energy, is mainly observed and compared with speaker personality. Pitch-change can be observed by calculating the autocorrelation between the length of $W$ and the delay intervals in the length of $d$ using the formula shown below. From the calculated values, repeated peak intervals can be determined as the pitch. ${ }^{16}$

$$
C_{x}(d)=\sum_{n=n_{0}}^{n_{0}+W} x(n) x(n-d)
$$

However, the pitch of an individual's voice is unique, and using an absolute value can lead to a misjudgment. Thus the level of pitch-change during speech may be more useful than an absolute value of pitch to analyze the correlation between pitchchange and user personality.

Since the introverted are generally more meticulous in their selection of communicated thoughts and vocabulary, it is advisable to calculate statistics on the frequencies and lengths of their silent intervals (silent periods in speech) for a more accurate analysis. ${ }^{8}$ The speech information of subjects is divided into silent intervals and vocal intervals, and the lengths of the intervals are calculated and converted as follows: 


$$
S_{\text {user }}=\left\{v_{1}, s_{1}, v_{2}, s_{2}, \ldots, s_{n-1}, v_{n}\right\}
$$

$\mathrm{v}$ : length of vocal intervals, s: length of silent intervals.

Three types of information are extracted from the data converted as shown above. The first type is how long the silent intervals are. The mean value and standard deviation of silent interval lengths of each subject are calculated:

$$
s_{\text {avg }}=\frac{\sum_{i=1}^{n-1} s_{i}}{n-1}, s_{s d}=\sqrt{\frac{\sum_{i=1}^{n-1}\left(s_{i}-s_{\text {avg }}\right)^{2}}{n-1}}
$$

The second type is a calculation of the ratio of silent intervals in a single total speech interval as follows:

$$
S R=\frac{\sum_{i=1}^{n-1} s_{i}}{\sum_{i=1}^{n} v_{i}+\sum_{i=1}^{n-1} s_{i}}
$$

The third type is the total number of silent intervals in a set duration. Silent intervals of more than an empirically pre-determined length are counted.

To analyze the relationship between silent intervals and user personality, we executed preliminary experiments with 20 extrovert students and 20 introvert students who were randomly selected in Sejong University, Seoul, Korea. The experiment discovered that user personality particularly influences the proportions of silent intervals and the frequency of long silences. The result was shown by boxplots. ${ }^{\dagger}$

Figure 2 shows a comparison of the proportions of silent intervals in speech. The test showed a noticeable difference between the proportions of silent intervals produced by introverted and extroverted subjects. From this finding, it was deduced that the high degree of freedom in the test resulted in the extroverted talking more and the introverted talking less. This is consistent with the findings of Dewaele ${ }^{8}$ which dictate that the extroverted are generally more fluent while the introverted are generally hesitant, and the findings of Furnham ${ }^{9}$ which dictate that while the extroverted are generally fluent in speech, the introverted generally think more in terms of conversations. Thus, this experiment confirmed that such features are important variables that can be used to identify introverted and extroverted individuals.

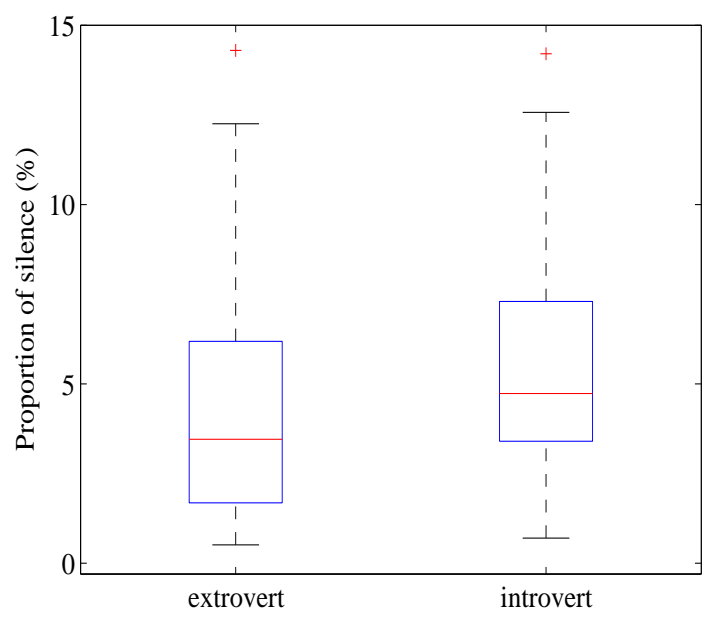

Fig. 2. Proportion of silent intervals in speech.(Source: findings from the preliminary experiments for this study)

Figure 3 shows a comparison of the numbers of silent intervals over a set length. In this experiment, the numbers of silent intervals longer than 2 seconds were added up for each subject. The numbers of silent intervals produced by extroverted/introverted subjects are shown. The average number of silent intervals produced by the extroverted was 1.1 and the number of silent intervals was usually between 0 and 3. However, the average number of silent intervals produced by the introverted was 3.2. This means that the introverted did not speak for a prolonged period more often than the extroverted and that the number of long silent intervals can also be used as an important variable to identify personalities due to reflecting hesitation.

\footnotetext{
${ }^{\dagger}$ A boxplot shows a box and whisker plot with one box. The boxes have lines at the lower quartile, median, and upper quartile values. The whiskers are lines extending from each end of the boxes to show the extent of the rest of the data. Outliers(red +) are data with values beyond the ends of the whiskers.
} 


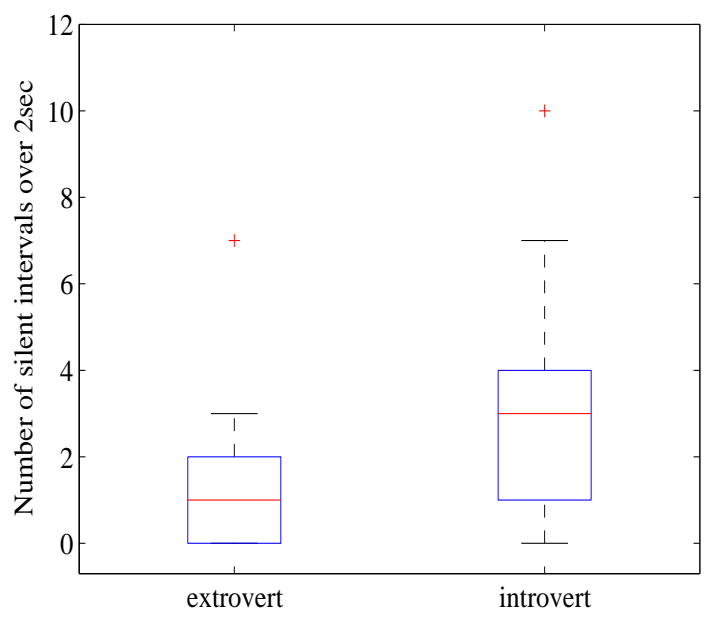

Fig. 3. Number of silent intervals in an extended duration(long silence).(Source: findings from the preliminary experiments for this study)

In addition, we took much interest in a specific silent interval, the length between the end of a question and the beginning of an answer, which we called "Reaction Time". It was supposed to be useful for classifying extroversion/introversion since the introverted are generally expected to take more time to collect and build their thoughts before speaking than the extroverted.[Figure 4]

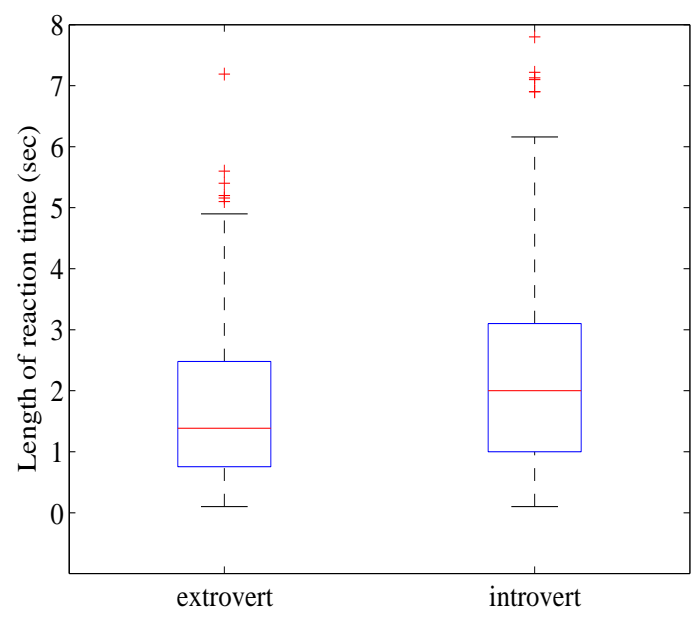

Fig. 4. Reaction time (Source: findings from the preliminary experiments for this study)
The various statistics regarding silent intervals were generally expected to differ by personality and were critically examined in this study. On the hypothesis that the initial section of a silent interval contains only background noise without voice and that background noise does not change suddenly while speaking takes place, a threshold was set based on the energy value in this section to examine silent intervals. Subsequently, sections with energy lower than the threshold can be classified as silent intervals. ${ }^{17}$ Although this method was not optimal, it was simple and effective enough for determining user personality trait.

\section{Pattern Classification Method}

The Support Vector Machine (SVM), which recently has been widely utilized in the field of machine learning, was used for user personality classification in this study. As the SVM provides a powerful kernel function that can identify the structural features of data, it is known to provide a more reliable estimation than the Multiple Discriminant Analysis (MDA), Logit, and Artificial Neural Network. ${ }^{18,19}$ In this study, C-Support Vector Classification (CSVC) was used, and the Radial Basis Function (RBF), designed to identify a non-linear relationship between a target value and input feature, was used as a kernel function. C-SVC solves the primal optimization problem shown below. While $\phi(x)$ projects $x$ in the higher dimensional space, $C$ performs the role of a regulation parameter. ${ }^{20}$

$$
\begin{gathered}
\min _{\omega, b, \xi} \frac{1}{2} \omega^{T} \omega+C \sum_{i=1}^{l} \xi_{i} \\
\text { subject to } y_{i}\left(\omega^{T} \phi\left(x_{i}\right)+b\right) \geqslant\left(1-\xi_{i}\right), \xi_{i} \geqslant 0, \\
i=1, \ldots l
\end{gathered}
$$

To improve the estimation accuracy of C-SVC, input vector values must be normalized between -1 and 1 or 0 and 1 , and a process of searching for values of $C$ and $\gamma$ that produce the highest estimation accuracy is required. In this study, the grid-search method was used to find the optimum values of $C$ and $\gamma$, and 5-fold cross-validation was used to find 
values that produced the highest estimation accuracy. A LIBSVM package was used for grid search analysis and C-SVC estimation. ${ }^{21}$

\section{Experiment and results}

To analyze user personalities and speech behaviors, a personality test was carried out on subjects as a preliminary test to determine their personalities. The personality test consisted of a survey consisting of 10 questions. ${ }^{22}$ Subjects who took part in the survey were male and female university students between 20 and 29 years of age. From a total of 138 subjects, 20 introverted subjects ( 1 to 5 points) and 20 extroverted subjects ( 8 to 10 points) were selected for a main test. To exclude subjects who were neither extroverted nor introverted (based on the survey results), only students who were either clearly extroverted or introverted were included in the second round of selection since subjects that even trained professionals would face difficulty in classifying were not compatible with the objective of this study. However, we also made sure there were an equal number of extroverted and introverted test subjects for the study. The reasoning behind having an equally number of extroverts and introverts was based on the goal of building an automated personality pattern classification system. The voice data from the subjects was needed to train the system and test its performance.

The main test carried out on the 40 subjects involved a question and answer session for the purpose of recording their voices in Korean. The questioner asked each subject two sets of 5 questions [Table 1] regarding their families and everyday life. The total of 80 sets of voice data ( 280 minutes) were used in the experiment. All voice data were set in sampling frequencies of $16 \mathrm{khz}$, mono channels, and 16bits. Data recording took place in a quiet laboratory environment.

A total of seven features sets were extracted from the acquired voice data. Table 2 describes the seven feature sets used in this experiment and their meanings. Speech rate (Speech speed), fundamental frequency for pitch, and energy were first extracted from voice data as a baseline. In addition, we ex- tracted the features related to silence (the critical subject of analysis in this research) such as silent interval lengths/ratios, long silent intervals, and the lengths of reaction time. Each feature set was normalized to a value between 0 and 1 . The purpose of data scaling is to avoid attributes in greater numeric ranges dominating those in smaller numeric ranges. The regulation allowed each variable to have an equal influence on the target variables.

Table 1. List of questions.(Source: findings from the preliminary study)

\begin{tabular}{|l|l|}
\hline set & Question \\
\hline \multirow{4}{*}{1} & $\begin{array}{l}\text { What is the most important thing in your } \\
\text { life? }\end{array}$ \\
\cline { 2 - 3 } & $\begin{array}{l}\text { Would you explain your life since your } \\
\text { childhood? }\end{array}$ \\
\cline { 2 - 2 } & Would you introduce your family? \\
\cline { 2 - 3 } & What do you like to do now and why? \\
\cline { 2 - 3 } & $\begin{array}{l}\text { What kind of role do you play in a group } \\
\text { of colleagues? }\end{array}$ \\
\hline \multirow{4}{*}{2} & What is your merit/weakness? \\
\cline { 2 - 2 } & What is your future plan? \\
\cline { 2 - 3 } & $\begin{array}{l}\text { What kind of difficulty have you } \\
\text { experienced? }\end{array}$ \\
\cline { 2 - 2 } & What do you think about your reputation? \\
\cline { 2 - 2 } & What is your hobby? \\
\hline
\end{tabular}

Table 2. Description of input variables.(Source: findings from the preliminary experiments for this study)

\begin{tabular}{|c|l|}
\hline Feature Set & Description \\
\hline F1 & Speech speed \\
\hline F2 & Pitch (Fundamental frequency) \\
\hline F4 & Energy \\
\hline F5 & $\begin{array}{l}\text { Lengths of silent intervals } \\
\text { Proportion of silent intervals } \\
\text { in total speech }\end{array}$ \\
\hline F6 & $\begin{array}{l}\text { Number of silent intervals } \\
\text { longer than a set length }\end{array}$ \\
\hline F7 & $\begin{array}{l}\text { Lengths of intervals between } \\
\text { the end of a question and } \\
\text { the beginning of an answer }\end{array}$ \\
\hline
\end{tabular}


To conduct an experiment that uses C-SVC, a kernel function and parameter must first be set. When using a linear kernel, only $C$ (penalty parameter) must be set; however, because the RBF nonlinear kernel is used in this experiment, the value of $\gamma$ was also required to be set. The optimum values of $C$ and $\gamma$ for each feature set were searched using the grid search method, which involves increasing the value of $C$ and $\gamma$ by 1 from -10 to 10 .

Figure 5 shows a comparison of the classification accuracy of the seven feature sets through 5fold cross validation. The average classification accuracy of energy was found to be $73.8 \%$. This finding can be attributed to the fact that the voices of the extroverted were generally louder. However, while the voices may have been physically louder, the fact that the extroverted are likely to be psychologically more active in their speech behavior may also have played a role. In this experiment, speaker personality was most accurately exhibited in the proportion of silent intervals in speech (F5) at an accuracy level of $76.3 \%$. The silence-related features of F4, F5, F6, and F7 also showed high levels of accuracy. Hence, speaker personality was best exhibited in various statistics on silence in natural speech. The test not only produced different responses from subjects but also enabled the subjects to exhibit personality-based tendencies in their responses to questions.

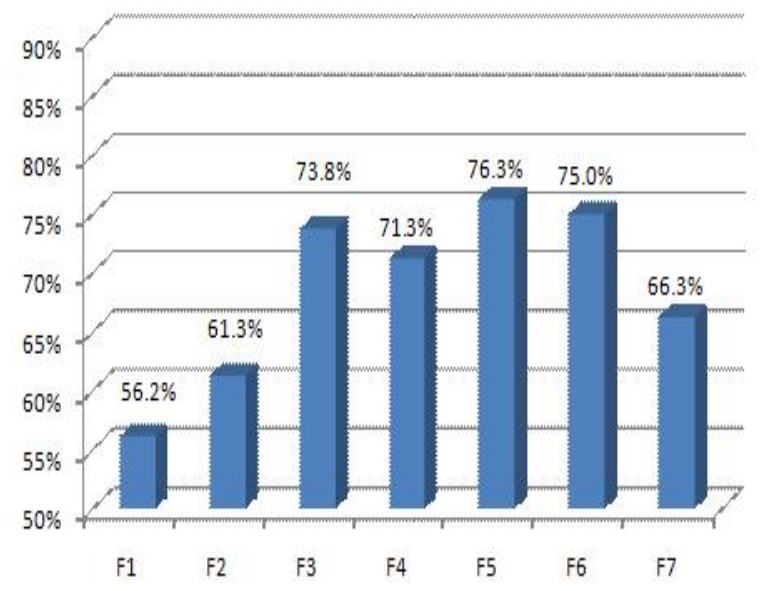

Fig. 5. Classification accuracy of each feature set.(Source: findings from the experiments of this study)
It seems that each feature of Table 2 plays a considerable role in personality trait classification. To increase the accuracy of classification we tried to analyze the relationship among those features (F1-F7) and classify personality traits with combined features. Since previous studies have shown that the speed, pitch, and energy of speech are points of reference for classifying the extroverted and the introvert, we regarded the combination of F1, F2, and $\mathrm{F} 3$ as a baseline. The experiment on the baseline showed that the accuracy went up to $82.5 \%$ [Figure 6]. Next we added the other features, F4, F5, F6, and F7, to the baseline in order. F5 and F6 improved the accuracy up to $85 \%$ and $83.8 \%$, respectively. However, F4 and F7 deteriorated the accuracy of classification. In the last step, we combined the baseline with F5 and F6. It resulted in the highest accuracy, 86.3\%.[Figure 6] It means that F5 and F6 supported the baseline for boosting up the classification accuracy (complementary relationship).

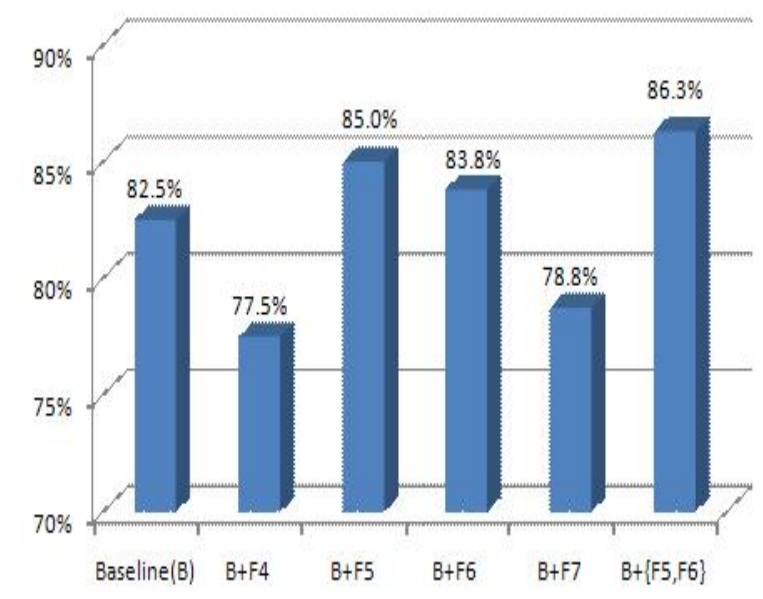

Fig. 6. Classification accuracy of each feature set.(Source: findings from the experiments of this study)

\section{Discussion}

The experimental results showed that our approach could be valid for user personality trait classification. However, it is doubtful whether the questions, 
which were used for speech data collection, properly reflected user personality or not. Therefore, a supplementary experiment was executed to assess the questions for their suitability. The test was to predict the personality traits attributed by human listeners to speakers who they listened to for the first time and who they were not acquainted with. It was carried out on 25 randomly selected subjects (13 males and 12 females). The personality test consisted of a survey of 20 listening questions. Subjects had to listen to the conversations which were user's answers to 10 questions, then made a decision, whether the user is extrovert or introvert. Also they chose their reasoning among five factors: speech speed, energy, pitch, silent intervals, and reaction time taken to reply a question.

The result showed that the average of classification accuracy was $86.6 \%$. It is similar to the accuracy achieved by our previous research, $86.3 \%$. Most of subjects (over 50\%) referred personality trait classification to speech energy and pitch [Figure 7] while silent intervals were the most powerful feature in the result of automatic personality trait classification by computer. The reason was analogically inferred that silent intervals are related with a quantitative analysis while the energy and pitch of speech is related with qualitative analysis. Humans may be unfamiliar with calculating silent intervals in the middle of listening user's speech.

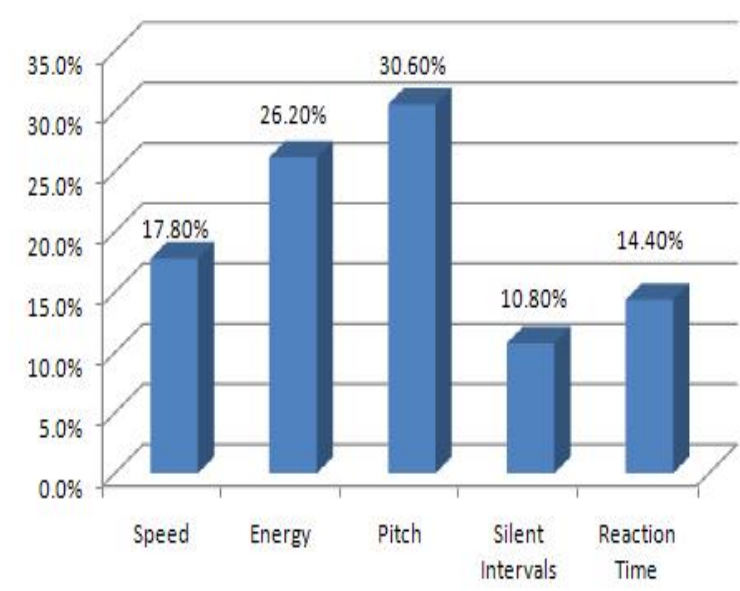

Fig. 7. Classification accuracy of each feature set by human.(Source: findings from the experiments of this study)
There was concern of bias creeping into the answers due in part to the type of question that was asked. We carried out another simple test to confirm if subjects had given biased answers. In this subsequent test, a transcript of the voice data that was devoid of any non-verbal cues was shown to 20 students who were not members of the final group. They were asked to analyze the transcripts and deduce whether the test subject was extroverted or introverted. Their accuracy was $32.5 \%$. This percentage is lower than a probability of $50 \%$ when using random selection. Therefore, this shows that classifying personality traits based on transcripts of voice data is a very difficult task. Conversely, it also shows that this results from this study are not biased from the standpoint of the type of questions. Furthermore, the accuracy rate of manual pattern classification reached $86.6 \%$ when non-verbal cues were presented along with the voice data taken from the discussion section. Overall, the content of the voice data as well as the linguistic information did not show any strong bias.

The objective of this study is to build a computer system that can analyze a person's personality as accurately as people are able to analyze personality traits of other people through one-on-one conversations. In the process used by us, only non-verbal cues were used to analyze personality traits in order to exclude the voice recognition processes. The exclusion of voice recognition makes it impossible to transcript the voice signals. Hence, natural language processing is excluded as well. The reason for excluding voice recognition is due to its computational resource demands, which will consume battery power rapidly. This would make it incompatible with smart devices and other next-generation computational devices. Focusing on non-verbal cues affords high automated performance when applied to extroversion/introversion classification and while this may be simplistic, it can be broadly used in many applications. However, this automated system has inherent limitations in analyzing a wide range of personalities.

The results from this study could be used to recognize a wide range of emotions. The factors 
that limit Emotion Recognition through voice data are individual and cultural characteristics, socioeconomic background, and differences in the way people express fleeting emotions. Thus, building an automated system for this type of task is too difficult. However, one's personality emerges subconsciously and while certain traits may be acquired temperament is usually determined at birth. We believe that if a common emotional response pattern shared among extrovert/introvert people is identifiable then the current system can be vastly improved through using extroversion/introversion classification in addition to the new process. This improved system could not only be deployed in smart devices but also in next-generation computing systems.

\section{Conclusions}

In this study, as well as the baseline such as the speed, pitch, and loudness of speech, various statistics on silent intervals were analyzed to determine the level of their contribution to personality trait classification by using actual data and an automatic decision-making algorithm. Consequently, it was discovered that user personality is strongly exhibited in the non-verbal cues of speech interface. It was also discovered that the proportion of silent intervals in speech produced when the subjects were answering questions is the most efficient non-verbal cue that can be used to identify speaker personality. In addition, speed, pitch, and energy in combination with some silent interval features achieved the accuracy of $86.3 \%$ for extroversion/introversion classification. In the future, new features, the type of questions, and any other non-verbal cue will require further study. Furthermore, it can be helpful for the extension of multi-modal approaches to study the effect of different modalities. The ability to recognize personality traits of users will be a necessary step towards natural-like man machine interaction, as promised by the vision of affective computing.

\section{Acknowledgments}

This research was supported by Basic Science Research Program through the National Research
Foundation of Korea (NRF) funded by the Ministry of Education, Science and Technology (No. 20110005334).

\section{References}

1. N. Caporusso, "Personality-Aware Interfaces for Learning Applications," In proceedings of ACM SIGUCCS Conference on University and College Computing Services, St. Louis, Missouri, USA, 189195 (2009).

2. M. Komarraju and S. J. Karau,"The relationship between the Big Five personality traits and academic motivation," Personality and Individual Differences, 39, 557-567 (2005).

3. F. Mairesse and M. Walker, "Automatic Recognition of Personality in Conversation," In proceedings of the Human Language Technology Conference of the North American Chapter of the ACL, 85-88 (2006).

4. S. Kwon, "Focused Word Spotting in Spoken Korean based on Fundamental Frequency," IEICE Electronics Express, 8(14), 1149-1154 (2011).

5. C.-M. Judd, L. James-Hawkins, V. Yzerbyt, and Y. Kashima, "Fundamental dimensions of social judgment: Unrdestanding the relations bteween judgments of competence and warmth," Journal of Personality and Social Psychology, 89(6), 899-913, (2005).

6. J.-M. Dewaele and A. Furnham, "Personality and speech production : a pilot study of second language learners," Personality and Individual Differences, 28, 355-365 (2000).

7. J.-B. Lee, "Influence of Personality Traits on Second Language Learning Based on the Five-Factor Model of Personality," Journal of English Language and Literature, 44, no.2, 209-237 (2000).

8. G. Matthews, I. J. Deary, and M. C. Whiteman, "Personality traits," Cambridge University Press, (2003).

9. A. Furnham, L. Forde, and T. Cotter, "Personaity and Intelligence," Personality and Individual Differences, 24, no.2, 187-192 (1998).

10. A. J. Gill and J. Oberlander, "Taking Care of the Linguistic Features of Extraversion," In proceedings of the 24th Annual Conference of the Cognitive Science Society, 363-368 (2002).

11. K. R. Scherer and U. Scherer, "Speech Behavior and Personality," Speech evaluation in psychiatry, New York, Grune \& Stratton, 115-135 (1981).

12. K. R. Scherer, "Personality inference from voice quality: the loud voice of extroversion," European Journal of Social Psychology, 8, 467-487 (1978).

13. W. Apple, L. A. Streeter, R. M. Krauss, "Effects of pitch and speech rate on personal attributions," Journal of Personality and Social Psychology, 37(5), 715727, (1979). 
14. M. H. Duncan, "An experimental study of some of the relationships between voice and personality among students of speech," Communication Monographs, (1945).

15. N. Morgan, E. Fosler, and N. Mirghafori, "Speech Recognition Using On-Line Estimation of Speaking Rate," In proceedings of Eurospeech, 4, 2079-2082 (1997).

16. A. M. Kondoz, "Digital Speech: Coding for Low Bit Rate Communication Systems," Second Edition, John Wiley \& Sons, Ltd., (2004).

17. S. Kwon, "Voice-Driven Sound Effect Manipulation," International Journal of Human-Computer Interaction, 28(6), 373-382 (2012).

18. Z. Huang, H. Chen, C.-J. Hsu, W.-H. Chen, and $\mathrm{S}$. $\mathrm{Wu}$, "Credit rating analysis with support vector machines and neural networks: a market comparative study," Decision Support Systems, 37, 543-558 (2004).

19. J. Kim, "Financial time series forecasting using support vector machines," Neurocomputing, 55, 307-319 (2003).

20. C. Cortes and V. Vapnik, "Support-vector network," Machine Learning, 20, 273-297 (1995)

21. C.-C. Chang and C.-J. Lin, "LIBSVM : a library for support vector machines," http://www.csie.ntu.edu.tw/ cjlin/libsvm, (2001).

22. B. Rammstedt and O. P. John, "Measuring personality in one minute or less: A 10-item short version of the Big Five Inventory in English and German,' Journal of Research in Personality, 41, 203-212, (2007) 